\title{
HOXD10 silencing suppresses human fibroblast-like synoviocyte migration in rheumatoid arthritis via downregulation of the $\mathrm{p38} / \mathrm{JNK}$ pathway
}

\author{
LUAN LUAN, YINGYING MA and LIHUA ZHANG \\ Department of Rheumatology, Jining No. 1 People's Hospital, Jining, Shandong 272000, P.R. China
}

Received December 13, 2017; Accepted March 29, 2018

DOI: $10.3892 /$ etm.2018.6432

\begin{abstract}
Homeobox D10 (HOXD10) belongs to the human homeobox (HOX) gene family, and the homologous protein encoded by HOX primarily controls cell differentiation and morphogenesis during embryonic development. The current study aimed to explore the roles and mechanisms of HOXD10 in the migration of human fibroblast-like synoviocytes in rheumatoid arthritis (RAFLS). Cell counting kit-8, cell migration and wound healing assays were performed to examine the cell viability and migration, respectively. Western blot and reverse transcription-quantitative polymerase chain reaction assays were used to evaluate the association between mRNA and protein expression levels. The results revealed HOXD10 expression was upregulated in tissues from patients with RA. HOXD10 silencing inhibited the viability of RAFLS. In addition, HOXD10 silencing suppressed the migration of RAFLS through modulating the expression of cadherin-11, N-cadherin, E-cadherin, vimentin, zonula occludens-1, integrin $\beta 1$ and paxillin. In conclusion, HOXD10 silencing downregulates the p38/c-Jun N-terminal kinase signaling pathway, which in turn may suppress the migration of RAFLS.
\end{abstract}

\section{Introduction}

Rheumatoid arthritis (RA), a chronic and systemic autoimmune disease, primarily affects the peripheral synovial joints and causes synovitis, which thereby damages the articular cartilage as well as bone tissues (1). Currently, on account of the irreversible joint damage and systemic osteoporosis, RA represents one of the most disabling diseases. It has been published that $0.5-1 \%$ of adults are affected by RA in

Correspondence to: Dr Lihua Zhang, Department of Rheumatology, Jining No. 1 People's Hospital, 6 Jiankang Road, Fuqiao Street, Jining, Shandong 272000, P.R. China

E-mail: lihuazhang29zhl@163.com

Key words: homeoboxD10, migration, rheumatoid arthritis, fibroblast-like synoviocytes, p38, c-Jun N-terminal kinase, cadherin-11, vimentin, integrin $\beta 1$ the developed world (2). It was reported that the incidence of RA in 2010 was 28.5 per 100,000 person-years in Korea (3). Additionally, RA is considered a chronic inflammatory disease and an autoimmune disease, due to the hyperplasia of synovial tissues in an inflammatory environment (4). Persistent and progressive development of inflammation in RA may develop into articular spasticity, deformity and dysfunction, which seriously affect the health and quality of life of patients with RA (5). The pathological mechanism of RA is complex, and basic pathological features are involved in systemic synovitis and formation of pannus. The immunopathology of pannus is divided into two parts: Immune and invasion (6-8). Osteoclasts and synovial fibroblasts (SFBs) are involved in the invasion. SFBs primarily consist of two types: Type A (macrophage-like) and type B (fibroblast-like). The number of fibroblast-like synoviocytes (FLS) is large, and their rich nuclear material suggests that these cells are in an active state of RNA metabolism (9). In addition, RAFLS represent a type of primary cell that can be cultured, proliferated, and passaged multiple times in vitro to maintain defined growth and invasion capacities, which is similar to tumor cells (10). Delays in the diagnosis and early treatment of RA lead to poor RA prognoses. Therefore, in order to improve the prognosis of RA patients, in-depth investigations into the underlying genetic or pathophysiological factors are necessary for developing effective treatments for RA.

Mitogen-activated protein kinases (MAPKs), a class of intracellular serine/threonine protein kinases located in the majority of cells, can transmit extracellular stimuli into intracellular signaling pathways leading to a series of cellular biological reactions, including cell differentiation, proliferation and apoptosis (11). In mammals, MAPKs are associated with four major pathways, including p38, c-Jun N-terminal kinase (JNK), extracellular signal-regulated kinases (ERKs), which contribute to the cellular responses to various stimuli, such as inflammatory cytokines, physical and chemical stress and bacterial products (12). It has been suggested that both p38 and JNK serve crucial roles in the biological activity of FLS in RA $(13,14)$. In addition, previous studies have demonstrated that the p38/JNK signaling pathway contributes to the modulation of migration in various cells, including smooth muscle cell subtypes (15), tumor cells (16-18) and stem cells (19). However, little is known about the roles and mechanisms of the p38/JNK signaling pathway in the migration of FLS in RA. 
Homeobox D10 (HOXD10), as a member of human homeobox (HOX) gene family, is associated with cell differentiation and morphogenesis during embryonic development $(20,21)$. Previous studies have demonstrated close associations between HOX gene families and the occurrence, development and prognosis of multiple malignant tumors $(22,23)$. In addition, HOX gene families have been demonstrated to participate in the regulation of tumor cell proliferation, differentiation, apoptosis via the adenomatous polyposis coli protein/ $\beta$-catenin signaling pathways $(24,25)$. Recently, it has been demonstrated that HOXD10 serves as a modulator in the migration of several cancer cell types (26-28). Nevertheless, the function of HOXD10 in the migration of RAFLS remains unclear. Therefore, HOXD10 was selected as the focus of this study to further explore its role and mechanisms in RA.

In the current study, the correlation between HOXD10 and the migration of RAFLS was analyzed. Furthermore, the exact role and mechanisms of HOXD10 within thep38/JNK signaling pathway were investigated with respect to the migration of RAFLS.

\section{Materials and methods}

Tissue samples. A total of 30 patients (age range, 37-60; male:female, 9:21) diagnosed with RA in the Jining No. 1 People's Hospital (Jining, China) from December 2014 to January 2016 were included in this study. Immediately following resection, all tissue samples were snap-frozen in liquid nitrogen and stored at $-80^{\circ} \mathrm{C}$. Matched adjacent normal synovium tissue was collected and used as a negative control. All patients included in the study provided written informed consent for the utilization of the tissue samples for clinical research. The project protocol was approved by the Institutional Review Board of Jining No. 1 People's Hospital.

Cell culture, gene, plasmid and grouping. Human RAFLS were separated from the RA knee-joint tissue samples of an RA patient selected at random. RAFLS were maintained in Dulbecco's modified Eagle's medium (DMEM; Gibco; Thermo Fisher Scientific, Inc., Waltham, MA, USA) supplemented with $10 \%$ fetal bovine serum (FBS; Gibco; Thermo Fisher Scientific, Inc.) in a $5 \% \mathrm{CO}_{2}$ atmosphere at $37^{\circ} \mathrm{C}$. The HOXD10 small interfering RNA (siRNA) was cloned into the empty pcDNA3.1 (+) vector (Invitrogen; Thermo Fisher Scientific, Inc.), further referred to as si-HOXD10 [this was a gift from Corey Largman (Addgene plasmid cat. no. 21007)]. Cells were divided into the following three treatment groups: Control group, untreated RAFLS; NC group, RAFLS transfected with empty vector; and siHOXD10 group, RAFLS transfected with si-HOXD10.

Cell viability analysis. A cell counting kit-8 (CCK-8; Beyotime Institute of Biotechnology, Shanghai, China) was used to study the viability of RAFLS. RAFLS in the logarithmic phase $\left(6 \times 10^{4}\right.$ cells $\left./ \mathrm{ml}\right)$ were seeded into the wells of 96 -well plates, and then incubated in $5 \% \mathrm{CO}_{2}$ atmosphere at $37^{\circ} \mathrm{C}$ for $12 \mathrm{~h}$. RAFLS were then divided into the three aforementioned treatment groups. The cells were maintained for 24,48 and $72 \mathrm{~h}$. A total of $10 \mu \mathrm{l}$ of CCK-8 reagent was then added to the wells.
Cells were maintained for a further $3 \mathrm{~h}$ at $37^{\circ} \mathrm{C}$. A microplate reader was used to record the absorbance at $450 \mathrm{~nm}$. Cell viability was quantified as the percentage of surviving cells relative to the control.

Wound healing assay. Cultured RAFLS were seeded in 24-well plates $\left(1 \times 10^{5}\right.$ cells $\left./ \mathrm{ml}\right)$ and divided into the three aforementioned treatment groups. RAFLS were then maintained for 15 days until the cell confluence reached $100 \%$. Horizontal 'wounds' were generated using a $10 \mu \mathrm{l}$ pipette tip scraping along the surface of each well. Cells were then washed with Hanks liquor (Beijing Solarbio Science \& Technology Co., Ltd., Beijing, China) three times to remove the loose cells and serum-free medium (Gibco; Thermo Fisher Scientific, Inc.) was then added. Pictures were obtained using an inverted microscope at 0 and $24 \mathrm{~h}$ time points.

Migration assay. Cells were transfected with an empty vector or siHOXD10. After $36 \mathrm{~h}$, RAFLS were digested using $0.25 \%$ trypsin (Gibco; Thermo Fisher Scientific, Inc.). Cells $\left(1 \times 10^{6} \mathrm{cells} / \mathrm{ml} ; 100 \mu \mathrm{l}\right)$ were plated in DMEM without serum in the top chamber of the Transwell insert (Corning Incorporated, Corning, NY, USA); while DMEM with 20\% FBS was added in the lower well. After $24 \mathrm{~h}$ of incubation at $37^{\circ} \mathrm{C}$, cells in the lower well were treated with $1 \mathrm{ml}$ paraformaldehyde $(4 \%)$ for $30 \mathrm{~min}$ at room temperature and subsequently stained with $0.1 \%$ crystal violet (Beijing Solarbio Science \& Technology Co., Ltd.) for $20 \mathrm{~min}$ at room temperature. Cells were washed three times with PBS and the optical density was recorded at $570 \mathrm{~nm}$ using a microplate reader.

Western blot analysis. The cells were seeded at a density of $1 \times 10^{3}$ cells/well into the 24 -well plates. Following the cell confluence reaching $65 \%$, the cells were transfected with empty vector or siHOXD10. After $36 \mathrm{~h}$, the proteins were isolated with NP40 lysis buffer (Beyotime Institute of Biotechnology). The concentration was estimated by a BCA kit provided by Bio-Rad Laboratories, Inc. (Hercules, CA, USA). The proteins ( $25 \mu \mathrm{g} /$ lane) were separated by $12 \%$ SDS-PAGE gels. The separated products were transferred to a PVDF membrane. The membranes were blocked with $5 \%$ non-skimmed milk at temperature for $2 \mathrm{~h}$ at room temperature. Blotting was performed with specific antibodies at $4^{\circ} \mathrm{C}$ overnight: Rabbit anti-human anti-HOXD10 (dilution, 1:1,000; cat. no. ab138508); anti-cadherin-11 (dilution, 1:500; cat. no. ab151302); anti-N-cadherin (dilution, 1:1,000; cat. no. ab76057); anti-E-cadherin (dilution, 1:500; cat. no. ab15148); anti-vimentin (dilution, 1:500; cat. no. ab137321); anti-zonula occludens-1 (Zo-1; dilution, 1:1,000; cat. no. ab216880); anti-integrin $\beta 1$ (dilution, 1:2,000; cat. no. ab179471); anti-paxillin (dilution, 1:500; cat. no. ab2264); anti-phosphorylated (p)-p38 (dilution, 1:1,000; cat. no. ab4822); anti-p38 (dilution, 1:2,000; cat. no. ab170099); anti-p-JNK (dilution, 1:2,000; cat. no. ab124956); anti-JNK (dilution, 1:1,000; cat. no. ab179461); anti-GAPDH (dilution, 1:2,500; cat. no. ab9485); anti- $\beta$-actin (dilution, 1:1,000; cat. no. ab8227; all Abcam, Cambridge, UK). The membranes were then incubated with horseradish peroxidase-conjugated secondary antibodies (goat anti-rabbit; dilution, 1:5,000; cat. no. ab205718; Abcam) at room temperature for $1 \mathrm{~h}$. Enhanced 
chemiluminescent stain (EMD Millipore, Billerica, MA, USA) was used to evaluate the results. The density of the bands were quantified with ImageQuant software (version 5.2; Molecular Dynamics; GE Healthcare, Chicago, IL, USA).

Reverse transcription-quantitative polymerase chain reaction (RT-qPCR) analysis. The cells were seeded at a density of $1 \times 10^{3}$ cells/well into the 24 -well plates. Following the cell confluence reaching $65 \%$, the cells were transfected with empty vector or siHOXD10. After $36 \mathrm{~h}$, total RNA was extracted from cultured RAFLS using TRIzol reagent (Invitrogen; Thermo Fisher Scientific, Inc.). RNA was reverse transcribed to cDNA using a Reverse Transcription kit (Beyotime Institute of Biotechnology) according to the manufacturer's instructions. RT-qPCR analysis was performed with an ABI 7500 Thermocycler (Applied Biosystems; Thermo Fisher Scientific, Inc.) PCR cycles were as followed: 4 min pretreatment at $95^{\circ} \mathrm{C}$; followed by 35 cycles of $95^{\circ} \mathrm{C}$ for $25 \mathrm{sec}$ and $60^{\circ} \mathrm{C}$ for $30 \mathrm{sec}$; then extension at $72^{\circ} \mathrm{C}$ for $10 \mathrm{~min}$. The samples were then maintained at $4^{\circ} \mathrm{C}$. The primers were designed by Invitrogen (Thermo Fisher Scientific, Inc.): HOXD10, forward, 5'-AAG ATGAACGAGCCCGTGAG-3', and reverse, 5'-TCCAGCGTT TGGTGCTTAGT-3', (product, 242 bp); cadherin-11, forward, 5'-GCCGAAGCCTACATCCTCAA-3', and reverse, 5'-TCA ACGCTATTGGGAGCTGG-3', (product, 332 bp); N-cadherin, forward, 5'-CCAAGTGAACGATAAGGGCG-3', and reverse, 5'-CCTTACTCTTGCCACCCTGA-3', (product, 209 bp); E-cadherin, forward, 5'-GTGAACACCTACAATGCC GC-3', and reverse, 5'-CAAAATCCAAGCCCGTGGTGG-3', (product, $274 \mathrm{bp}$ ); vimentin, forward, 5'-AATAAGATCCTG CTGGCCGA-3', and reverse, 5'-GGTGTTTTCGGCTTCCTC TC-3', (product, 225 bp); Zo-1, forward, 5'-GGAGGTAGA ACGAGGCATCA-3', and reverse, 5'-AGGCCTCAGAAA TCCAGCTT-3', (product, 238 bp); integrin $\beta 1$, forward, 5'-TCC AACCTGATCCTGTGTCC-3', and reverse, 5'-CAATTCCAG CAACCACACCA-3', (product, $174 \mathrm{bp}$ ); paxillin, forward, 5'-ATCCTGAGTGCTTTGTGTGC-3', and reverse, 5'-CCT TGTTGAGCTGCTTGAGG-3', (product, 225 bp); GAPDH, forward, 5'-GAGTCCACTGGCGTCTTCA-3', and reverse, 5'-GGTCATGAGTCCTTCCACGA-3', (product, 240 bp); $\beta$-actin, forward, 5'-GTTACAGGAAGTCCCTCACCC-3', and reverse, 5'-CAGACCTGGGCCATTCAGAAA-3', (product, $194 \mathrm{bp})$. GAPDH and $\beta$-actin were used as the endogenous controls. The $2^{-\Delta \Delta \mathrm{Cq}}$ method was used for the calculation of relative expression (29).

Statistical analysis. The results are reported as the mean \pm standard error of the mean of at least three independent experiments. All the experimental data was analyzed by paired Student's t-test, or one-way analysis of variance followed by a Tukey's test. The statistical significance was defined as $\mathrm{P}<0.05$.

\section{Results}

HOXD10 expression is upregulated in tissues from patients with $R A$. RT-qPCR analysis was performed to examine the expression of HOXD10 in human RA tissue samples and matched adjacent normal synovium tissues of the knee-joint from four patients with RA, selected at random. It was revealed that the expression of HOXD10 in RA tissues was significantly higher compared with that observed in the matched adjacent normal tissues $(\mathrm{P}<0.01$; Fig. 1A). RT-qPCR and western blot analyses were performed to evaluate the HOXD10 protein expression in four random paired human RA and normal adjacent synovium knee-joint tissues. The results indicated that the HOXD10 expression was significantly enhanced in RA tissue compared with the control sample $(\mathrm{P}<0.001$; Fig. 1B). This suggests that HOXD10 expression may be upregulated in RA tissue.

Identification of RAFLS. The SFBs were isolated from RA knee-joint tissue samples of a single patient with RA selected at random. During the course of the experiments, SFBs were cultured in DMEM medium containing 10\% FBS. Following $24 \mathrm{~h}$ in cell culture, the SFBs were studied using an inverted microscope. The adherent cells were SFBs. As shown in Fig. 2A, the morphology of SFBs was spindle-shaped, stellate and polygonal. The boundaries of nuclei were clear and oval-shaped. The nucleus was located in the middle of cells, and the nucleolus was clear. At passage four, FLS accounted for more than $98 \%$ of the total SFBs (Fig. 2B), which were harvested and used in subsequent studies.

Interference of HOXD10 in RAFLS. An siRNA vector targeting HOXD10, si-HOXD10, was constructed in the present study. RAFLS separated from RA patients were transfected with the pcDNA3.1 (+) empty vector or si-HOXD10. The knockdown efficiency was $\sim 90 \%$ in RAFLS following stable transfection with si-HOXD10 ( $<<0.001$; Fig. 3A). Western blot analysis demonstrated that following transfection with si-HOXD10, the expression level of HOXD10 was significantly reduced $(\mathrm{P}<0.001$; Fig. 3B).

HOXD10 silencing inhibits the viability of RAFLS. A CCK-8 assay was performed to measure the viability of RAFLS divided into the three aforementioned treatment groups. The results demonstrated that, compared with the control groups, HOXD10 silencing inhibited the viability of RAFLS, particularly at the 48 and $72 \mathrm{~h}$ time points $(\mathrm{P}<0.05$; Fig. $3 \mathrm{C})$.

HOXD10 silencing suppresses the migration of RAFLS. The migration capacity of RAFLS was evaluated using wound healing and migration assays. The wound healing assay results revealed that the wound width of RAFLS transfected with si-HOXD10 was significantly increased compared with the NC group ( $\mathrm{P}<0.01$; Fig. 4A). A similar trend was observed from the migration assay where the number of migrated RAFLS transfected with si-HOXD10 decreased from 100 to $60.3 \%$ ( $\mathrm{P}<0.05$; Fig. 4B). These results indicate, that HOXD10 silencing may decrease the migration ability of RAFLS.

HOXD10 silencing modulates the expression of migration-associated proteins in RAFLS. Considering the results of the migration assay, migration-associated mechanisms in RAFLS and the expression levels of migration-associated proteins, includingcadherin-11, N-cadherin, E-cadherin, vimentin, Zo-1, integrin $\beta 1$ and paxillin were assessed. On the basis of the RT-qPCR results, it was revealed that HOXD10 silencing significantly inhibited the expression levels of cadherin-11, E-cadherin, Zo-1, integrin $\beta 1$ and paxillinin 

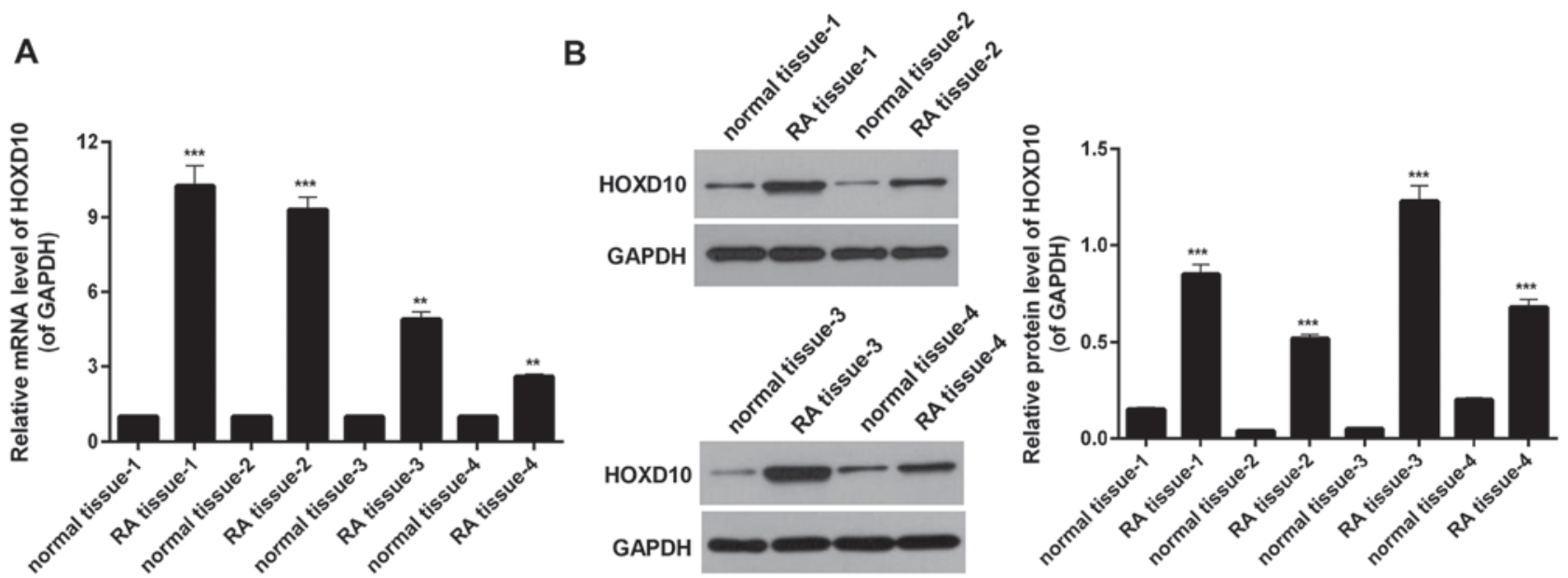

Figure 1. HOXD10 expression is upregulated in RA tissues from patients with RA. (A) Reverse transcription-quantitative polymerase chain reaction and (B) western blot assays were performed to examine the expression of HOXD10 in RA tissues and matched adjacent normal knee-joint tissues from RA patients. ${ }^{* *} \mathrm{P}<0.01$ and ${ }^{* * *} \mathrm{P}<0.001$ vs. normal tissue. HOXD10, homeobox D10; RA, rheumatoid arthritis.

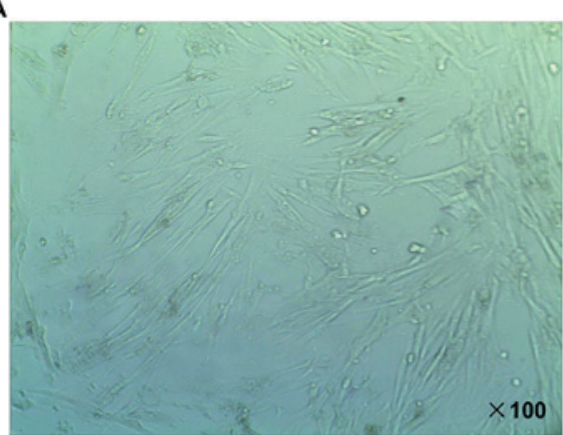

B

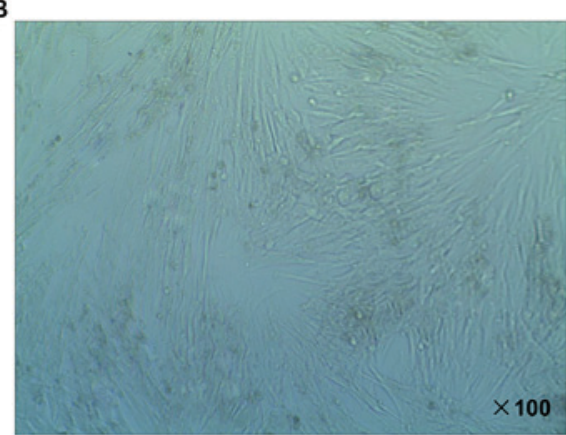

Figure 2. Identification of RAFLS. (A) Primary RAFLS cultures and (B) RAFLS at passage four were observed by inverted microscopy. Magnification, x100. RAFLS, fibroblast-like synoviocytes in rheumatoid arthritis.

A

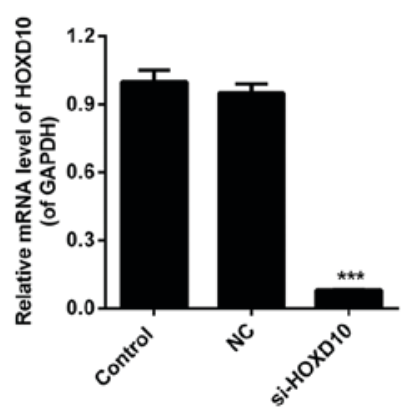

B

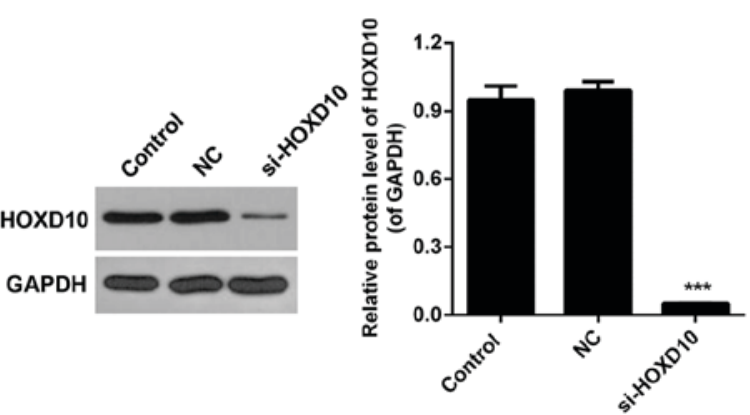

C

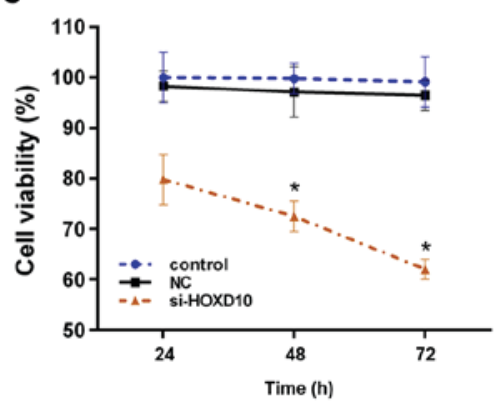

Figure 3. Interference of HOXD10 expression in RAFLS and its effect on cells. RAFLS were transfected with empty vector or with si-HOXD10. (A) Reverse transcription-quantitative polymerase chain reaction and (B) western blot assays were performed to measure the expression of HOXD10 in RAFLS. (C) A CCK-8 assay was performed to measure the viability of RAFLS. "P<0.05 and ${ }^{* * * *} \mathrm{P}<0.001$ vs. NC. HOXD10, homeobox D10; RAFLS, fibroblast-like synoviocytes in rheumatoid arthritis; si-HOXD10, small interfering RNA targeting HOXD10; CCK-8, Cell Counting Kit-8; NC, negative control.

RAFLS ( $\mathrm{P}<0.01$; Fig. 5A). In addition, it was observed that $\mathrm{N}$-cadherin and vimentin expression in RAFLS was significantly upregulated following transfection with si-HOXD10 $(\mathrm{P}<0.001$; Fig. 5A). Western blot analysis revealed similar trends for migration-associated protein expression in RAFLS (Fig. 5B and C). Based on these observations, it was identified that HOXD10 silencing may suppress the migration of RAFLS through modulating the expression levels of cadherin-11, $\mathrm{N}$-cadherin, E-cadherin, vimentin, Zo-1, integrin $\beta 1$ and paxillin.

HOXD10 silencing downregulates the p38/JNK signaling pathway. In order to further investigate the association between HOXD10 and the p38/JNK signaling pathway in RA, the expression levels of p-p38, p38, p-cJNK and JNK 
A
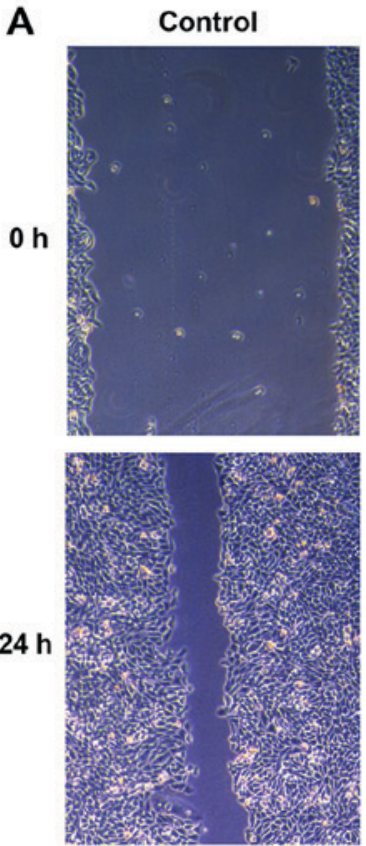

B

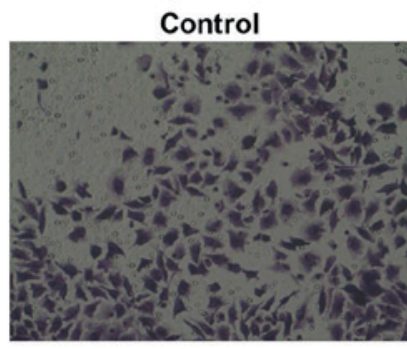

NC
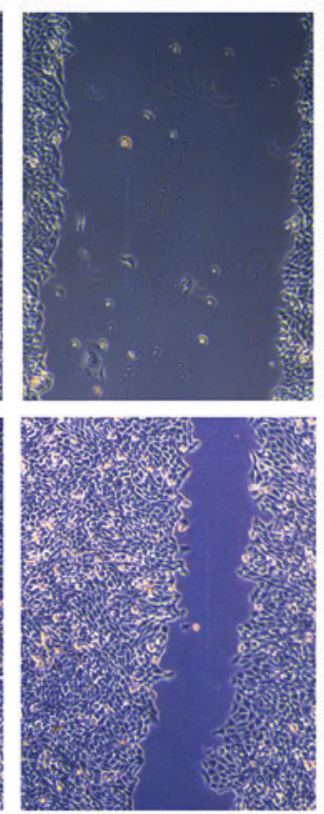
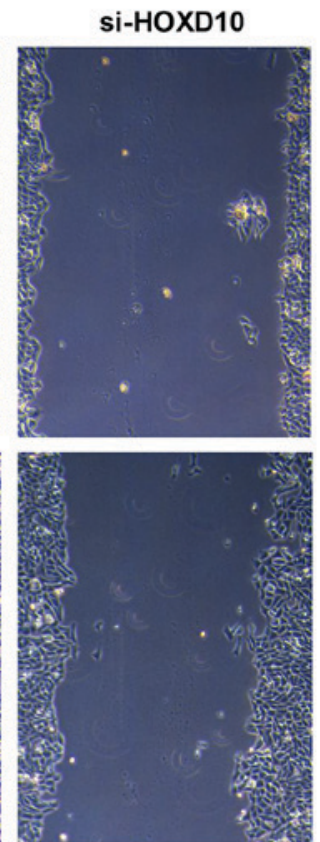

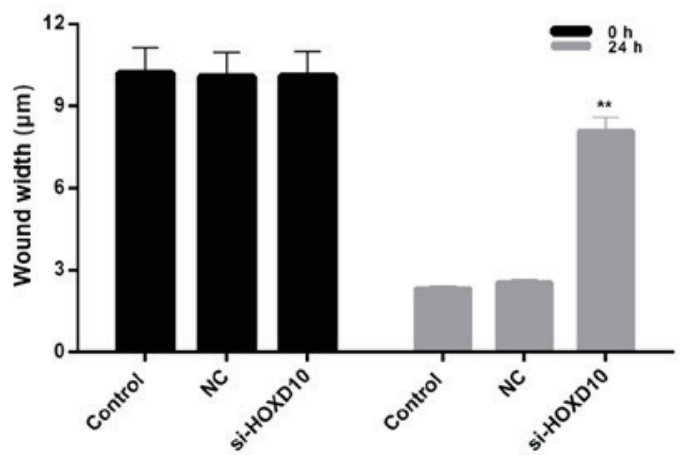

NC
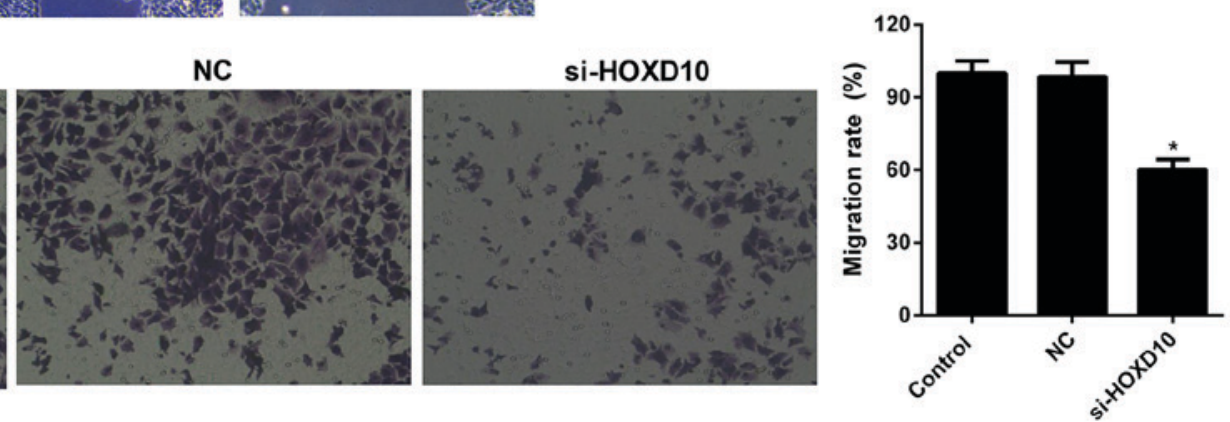

Figure 4. HOXD10 silencing suppresses the migration of RAFLS. (A) Wound healing and (B) migration assays were performed to evaluate the migration ability of RAFLS, RAFLS transfected with empty vector or si-HOXD10. ${ }^{*} \mathrm{P}<0.05$ and ${ }^{* *} \mathrm{P}<0.01$ vs. NC. HOXD10, homeobox D10; RAFLS, fibroblast-like synoviocytes in rheumatoid arthritis; si-HOXD10, small interfering RNA targeting HOXD10; NC, negative control.

in RAFLS were evaluated. Western blot analysis indicated that the expression levels of p-p38 and p-JNK in RAFLS were significantly downregulated following transfection with si-HOXD10 ( $\mathrm{P}<0.05$; Fig. 6). Therefore, HOXD10 silencing was demonstrated to suppress the phosphorylation of $\mathrm{p} 38$ and JNK in RAFLS. By contrast, there was no significant difference in $\mathrm{p} 38$ and JNK expression in RAFLS among each experimental group (Fig. 6). Therefore, it was concluded that HOXD10 silencing may affect the p38/JNK signaling pathway in RAFLS.

\section{Discussion}

RA is a chronic autoimmune disease with unknown etiology, which seriously endangers human health and life due to increased morbidity and the early age of onset (30). A crucial pathological feature of RA is chronic inflammatory lesions of synovium of joints, which destroys the articular cartilage and bone tissue. FLS are normally present in the synovial lining, but abnormal proliferation occurs in the FLS of patients with RA and gradually erodes the adjacent cartilage and bone (9). Although increased RAFLS in the synovial lining has been observed in situ, the index of enhancement of RA synovial membrane was not sufficient to account for the observed extent of synovial hyperplasia $(31,32)$. However, FLS from these tissues have been activated sustainably in vitro, with increased proliferative capacity and the ability to express a variety of growth factors, inflammatory cytokines, oncogenes and cyclins, thus exhibiting a tumor-like proliferation (33). Recently, studies have demonstrated that the FLS migration may also represent an important source of synovial hyperplasia. For example, it has been demonstrated that CA074Me, an inhibitor of cathepsin B, suppressed the migration and invasion of FLS in inflamed tissues of patients with RA (34). Therefore, inhibition of RAFLS migration may serve as a pivotal target for RA therapies.

Previous studies have suggested that HOXD10, a well-known tumor suppressor gene, serves a crucial role in the suppression of migration of various tumor cells (26-28), while the potential functions of HOXD10 in RAFLS migration remain unclear. Therefore, the aim of the present study was to investigate the involvement of HOXD10 in the suppression of RAFLS migration, and the associated mechanisms. Four pairs of human RA tissues and matched adjacent normal synovium tissues of knee-joints were randomly selected from RA patients and HOXD10 expression in these tissue samples was measured. According to the RT-qPCR and western blot results, it was revealed that the expression levels of HOXD10 


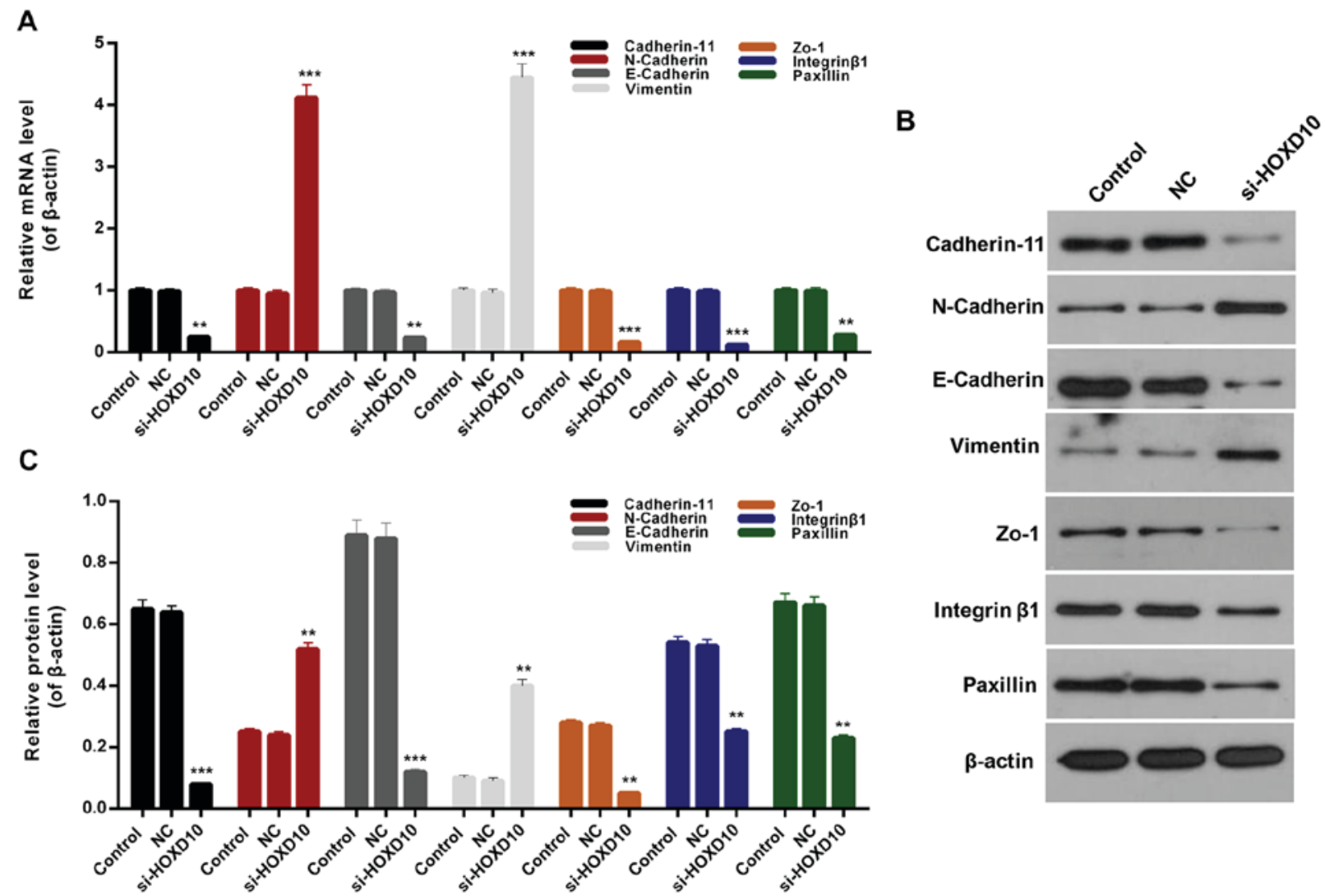

Figure 5. HOXD10 silencing affects the expression of migration-associated proteins. (A) Reverse transcription-quantitative polymerase chain reaction and (B) western blot assays were performed to determine the expression levels of cadherin-11, N-cadherin, E-cadherin, vimentin, Zo-1, integrin $\beta 1$ and paxillin in RAFLS, RAFLS transfected with empty vector or si-HOXD10. (C) Quantified western blotting results. ${ }^{* *} \mathrm{P}<0.01$ and ${ }^{* * * *} \mathrm{P}<0.001$ vs. NC. HOXD10, homeobox D10; Zo-1, zonula occludens-1; RAFLS, fibroblast-like synoviocytes in rheumatoid arthritis; si-HOXD10, small interfering RNA targeting HOXD10; $\mathrm{NC}$, negative control.

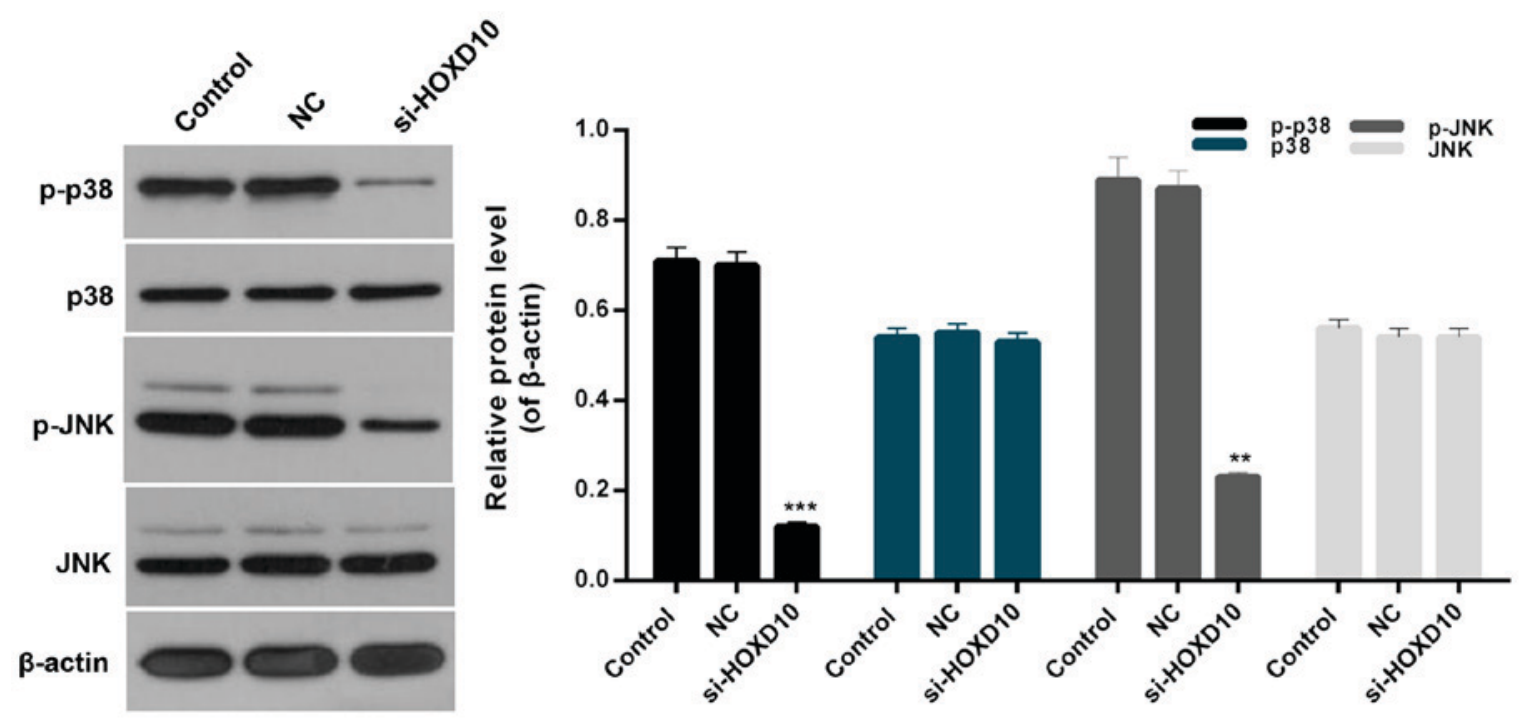

Figure 6. HOXD10 silencing downregulates the p38/JNK signaling pathway. Western blot assays were performed to measure the expression levels of p-p38, p38, p-JNK and JNK in RAFLS, RAFLS transfected with empty vector or si-HOXD10. ${ }^{* *} \mathrm{P}<0.01$ and ${ }^{* * * *} \mathrm{P}<0.001$ vs. NC. HOXD10, homeobox D10; JNK, c-Jun N-terminal kinase; p-p38, phosphorylated p38; p-JNK, phosphorylated JNK; RAFLS, fibroblast-like synoviocytes in rheumatoid arthritis; si-HOXD10, small interfering RNA targeting HOXD10; NC, negative control.

in RA tissues were significantly higher compared with those in the control tissue. Consequently, HOXD10 may serve as an important target in RA therapies. An siRNA vector targeting HOXD10 was constructed in this investigation and was used to transfect RAFLS, with the empty vector as a negative control. The knockdown efficiency was $~ 90 \%$ in RAFLS following stable transfection with si-HOXD10. The viability of RAFLS transfected with empty vector and si-HOXD10 was further 
assessed. It was revealed that HOXD10 silencing significantly suppressed the viability of RAFLS, particularly at $72 \mathrm{~h}$ following transfection. Moreover, the migration capacity of RAFLS transfected with empty vector and si-HOXD10 was evaluated by wound healing and Transwell assays. Based on the results, it was revealed that HOXD10 silencing significantly reduced the migration capacity of RAFLS. In order to investigate the involvement of HOXD10 in the migration of RAFLS further, the expression of several migration-associated proteins were measured. $\mathrm{N}$-cadherin, E-cadherin and vimentin are the most common markers used to evaluate cellular migration abilities $(35,36)$. In addition, cell adhesion is associated with cell migration $(37,38)$. Therefore, specific cell adhesion-associated molecules were selected to assess cell migration in the present study, including Zo-1, integrin $\beta 1$ and paxillin. It was revealed that HOXD10 silencing significantly downregulated the expression levels of cadherin-11, E-cadherin, Zo-1, integrin $\beta 1$ and paxillin, while it enhanced the expression of $\mathrm{N}$-cadherin and vimentin in RAFLS. Collectively, these results suggested that HOXD10 silencing may suppress the migration of RAFLS through influencing the expression levels of cadherin-11, N-cadherin, E-cadherin, vimentin, Zo-1, integrin $\beta 1$ and paxillin.

The p38/JNK signaling pathway has been demonstrated to participate in cell migration processes $(39,40)$. However, the association between the $\mathrm{p} 38 / \mathrm{JNK}$ signaling pathway and RAFLS migration was not very clear. In the current study, the expression levels of p-p38, p38, p-JNK and JNK in RAFLS transfected with empty vector and si-HOXD10 were evaluated. The results indicated that HOXD10 silencing reduced the phosphorylation of p38 and JNK in RAFLS, whereas no significant difference was detected in p38 and JNK expression in RAFLS among all groups. These results indicated that HOXD10 silencing affected the p38/JNK signaling pathway in RAFLS.

In conclusion, the results of the present study demonstrated that HOXD10 silencing inhibited the migration of RAFLS potentially via the $\mathrm{p} 38 / \mathrm{JNK}$ signaling pathway. The results provide valuable insight into the mechanisms of HOXD10 and FLS. The potential effects of HOXD10 on the migration of RAFLS suggest that HOXD10 may presentan effective target for RA therapies.

\section{Acknowledgements}

Not applicable.

\section{Funding}

No funding was received.

\section{Availability of data and materials}

All data generated and/or analyzed during the present study are included in this published article.

\section{Authors' contributions}

LL wrote the main manuscript. LL and YM performed the experiments. LZ designed the study. All authors read and approved the final manuscript.

\section{Ethics approval and consent to participate}

The present study was approved by the Ethics Committee of Jining No. 1 People's Hospital. Informed consent was obtained from the patients.

\section{Patient consent for publication}

Written informed consent was obtained from all participants for the publication of their data.

\section{Competing interests}

The authors declare that they have no competing interests.

\section{References}

1. Smolen JS, Aletaha D, Koeller M, Weisman MH and Emery P: New therapies for treatment of rheumatoid arthritis. Lancet 370: 1861-1874, 2007.

2. Smolen JS, Aletaha D and McInnes IB: Rheumatoid arthritis. Lancet 388: 2023-2038, 2016.

3. Won S, Cho SK, Kim D, Han M, Lee J, Jang EJ, Sung YK and Bae SC: Update on the prevalence and incidence of rheumatoid arthritis in Korea and an analysis of medical care and drug utilization. Rheumatol Int 38: 649-656, 2018.

4. Feldmann M, Brennan FM, Foxwell BM and Maini RN: The role of TNF alpha and IL-1 in rheumatoid arthritis. Curr Dir Autoimmun 3: 188-199, 2001

5. Pugner KM, Scott DI, Holmes JW and Hieke K: The costs of rheumatoid arthritis: An international long-term view. Semin Arthritis Rheum 29: 305-320, 2000.

6. Brennan FM, Hayes AL, Ciesielski CJ, Green P, Foxwell BM and Feldmann M: Evidence that rheumatoid arthritis synovial T cells are similar to cytokine-activated T cells: Involvement of phosphatidylinositol 3-kinase and nuclear factor kappaB pathways in tumor necrosis factor alpha production in rheumatoid arthritis. Arthritis Rheum 46: 31-41, 2002.

7. Lutzky V, Hannawi S and Thomas R: Cells of the synovium in rheumatoid arthritis. Dendritic cells. Arthritis Res Ther 9: 219, 2007.

8. Weyand CM, Kurtin PJ and Goronzy JJ: Ectopic lymphoid organogenesis: A fast track for autoimmunity. Am J Pathol 159: 787-793, 2001

9. Bartok B and Firestein GS: Fibroblast-like synoviocytes: Key effector cells in rheumatoid arthritis. Immunol Rev 233: 233-255, 2010.

10. Huber LC, Distler O, Tarner I, Gay RE, Gay S and Pap T: Synovial fibroblasts: Key players in rheumatoid arthritis. Rheumatology (Oxford) 45: 669-675, 2006.

11. Cuschieri J and Maier RV: Mitogen-activated protein kinase (MAPK). Crit Care Med 33 (12 Suppl): S417-S419, 2005.

12. Johnson GL and Lapadat R: Mitogen-activated protein kinase pathways mediated by ERK, JNK, and p38 protein kinases. Science 298: 1911-1912, 2002.

13. Inoue T, Hammaker D, Boyle DL and Firestein GS: Regulation of p38 MAPK by MAPK kinases 3 and 6 in fibroblast-like synoviocytes. J Immunol 174: 4301-4306, 2005.

14. Inoue T, Hammaker D, Boyle DL and Firestein GS: Regulation of JNK by MKK-7 in fibroblast-like synoviocytes. Arthritis Rheum 54: 2127-2135, 2006.

15. Kavurma MM and Khachigian LM: ERK, JNK, and p38 MAP kinases differentially regulate proliferation and migration of phenotypically distinct smooth muscle cell subtypes. J Cell Biochem 89: 289-300, 2003.

16. Liu QR, Liu JM, Chen Y, Xie XQ, Xiong XX, Qiu XY, Pan F, Liu D, Yu SB and Chen XQ: Piperlongumine inhibits migration of glioblastoma cells via activation of ROS-dependent p38 and JNK signaling pathways. Oxid Med Cell Longev 2014: 653732, 2014.

17. Ma CY, Ji WT, Chueh FS, Yang JS, Chen PY, Yu CC and Chung JG: Butein inhibits the migration and invasion of SK-HEP-1 human hepatocarcinoma cells through suppressing the ERK, JNK, p38, and uPA signaling multiple pathways. J Agric Food Chem 59: 9032-9038, 2011. 
18. Song $\mathrm{H}$ and Moon A: Glial cell-derived neurotrophic factor (GDNF) promotes low-grade Hs683 glioma cell migration through JNK, ERK-1/2 and p38 MAPK signaling pathways. Neurosci Res 56: 29-38, 2006.

19. Kuang D, Zhao X, Xiao G, Ni J, Feng Y, Wu R and Wang G: Stem cell factor/c-kit signaling mediated cardiac stem cell migration via activation of p38 MAPK. Basic Res Cardiol 103: 265-273, 2008.

20. Botas J: Control of morphogenesis and differentiation by HOM/Hox genes. Curr Opin Cell Biol 5: 1015-1022, 1993.

21. Samuel S and Naora H: Homeobox gene expression in cancer: Insights from developmental regulation and deregulation. Eur J Cancer 41: 2428-2437, 2005.

22. Grier DG, Thompson A, Kwasniewska A, Mcgonigle GJ, Halliday HL and Lappin TR: The pathophysiology of HOX genes and their role in cancer. J Pathol 205: 154-171, 2005.

23. Cantile M, Pettinato G, Procino A, Feliciello I, Cindolo L and Cillo C: In vivo expression of the whole HOX gene network in human breast cancer. Eur J Cancer 39: 257-264, 2003.

24. Jung C, Kim RS, Zhang H, Lee SJ, Sheng H, Loehrer PJ, Gardner TA, Jeng MH and Kao C: HOXB13 is downregulated in colorectal cancer to confer TCF4-mediated transactivation. Br J Cancer 92: 2233-2239, 2005.

25. Shah N and Sukumar S: The Hox genes and their roles in oncogenesis. Nat Rev Cancer 10: 361-371, 2010.

26. Nakayama I, Shibazaki M, Yashima-Abo A, Miura F, Sugiyama T, Masuda T and Maesawa C: Loss of HOXD10 expression induced by upregulation of miR-10b accelerates the migration and invasion activities of ovarian cancer cells. Int J Oncol 43: 63-71, 2013.

27. Wang YY, Li L, Ye ZY, Zhao ZS and Yan ZL: MicroRNA-10b promotes migration and invasion through Hoxd10 in human gastric cancer. World J Surg Oncol 13: 259, 2015.

28. Xiao H, Li H, Yu G, Xiao W, Hu J, Tang K, Zeng J, He W, Zeng $\mathrm{G}, \mathrm{Ye} \mathrm{Z}$ and $\mathrm{Xu} \mathrm{H}$ : MicroRNA-10b promotes migration and invasion through KLF4 and HOXD10 in human bladder cancer. Oncol Rep 31: 1832-1838, 2014.

29. Livak KJ and Schmittgen TD: Analysis of relative gene expression data using real-time quantitative PCR and the 2(-Delta Delta $\mathrm{C}(\mathrm{T})$ ) method. Methods 25: 402-408, 2001.

30. Gibofsky A: Overview of epidemiology, pathophysiology, and diagnosis of rheumatoid arthritis. Am J Manag Care 18 (13 Suppl): S295-S302, 2012.

31. Henderson B and Pettipher ER: The synovial lining cell: Biology and pathobiology. Semin Arthritis Rheum 15: 1-32, 1985.
32. Zvaifler NJ: Relevance of the stroma and epithelial-mesenchymal transition (EMT) for the rheumatic diseases. Arthritis Res Ther 8: 210, 2006

33. Firestein GS: Invasive fibroblast-like synoviocytes in rheumatoid arthritis. Passive responders or transformed aggressors? Arthritis Rheum 39: 1781-1790, 1996.

34. Tong B, Wan B, Wei Z, Wang T, Zhao P, Dou Y, Lv Z, Xia Y and Dai Y: Role of cathepsin $\mathrm{B}$ in regulating migration and invasion of fibroblast-like synoviocytes into inflamed tissue from patients with rheumatoid arthritis. Clin Exp Immunol 177: 586-597, 2014.

35. Nijkamp MM, Span PN, Hoogsteen IJ, van der Kogel AJ, Kaanders $\mathrm{JH}$ and Bussink $\mathrm{J}$ : Expression of E-cadherin and vimentin correlates with metastasis formation in head and neck squamous cell carcinoma patients. Radiother Oncol 99: 344-348, 2011.

36. Zhang X, Liu G, Yi K, Dong Z, Qian Q and Ma X: N-cadherin expression is associated with acquisition of EMT phenotype and with enhanced invasion in erlotinib-resistant lung cancer cell lines. PLoS One 8: e57692, 2013.

37. Newgreen DF and Gooday D: Control of the onset of migration of neural crest cells in avian embryos. Role of $\mathrm{Ca}++-$ dependent cell adhesions. Cell Tissue Res 239: 329-336, 1985.

38. Clay MR and Halloran MC: Regulation of cell adhesions and motility during initiation of neural crest migration. Curr Opin Neurobiol 21: 17-22, 2011.

39. Chen HJ, Lin CM, Lee CY, Shih NC, Peng SF, Tsuzuki M, Amagaya S, Huang WW and Yang JS: Kaempferol suppresses cell metastasis via inhibition of the ERK-p38-JNK and AP-1 signaling pathways in U-2 OS human osteosarcoma cells. Oncol Rep 30: 925-932, 2013.

40. Desai S, Laskar S and Pandey BN: Autocrine IL-8 and VEGF mediate epithelial-mesenchymal transition and invasiveness via p38/JNK-ATF-2 signalling in A549 lung cancer cells. Cell Signal 25: 1780-1791, 2013

C) (B) This work is licensed under a Creative Commons Attribution-NonCommercial-NoDerivatives 4.0 International (CC BY-NC-ND 4.0) License. 